10th World Online Conference on Soft Computing in Industrial Applications, Sep 2004,

Springer Series: Applications of Soft Computing : Recent Trends Series: Advances in Soft Computing , Vol. 36,

Tiwari, A.; Knowles, J.; Avineri, E.; Dahal, K.; Roy, R.; Editors

\title{
DEVELOPMENT OF FUZZY EXPERT SYSTEM FOR CUSTOMER AND SERVICE ADVISOR CATEGORISATION WITHIN CONTACT CENTRE ENVIRONMENT
}

\author{
Satya Shah; Rajkumar Roy; Ashutosh Tiwari. \\ \{s.shah, r.roy, a.tiwari\}@ cranfield.ac.uk \\ Enterprise Integration \\ Cranfield University, Cranfield \\ Bedfordshire. MK43 0AL \\ UK
}

\begin{abstract}
In this paper, we describe the research and development of a fuzzy expert system methodology for categorising customer and customer service advisor (CSA) within customer contact centre (CCC) environment. On the basis of data collected through case studies carried out within customer contact centre, two step clustering analysis within SPSS was used to derive the categories for customers and advisors based on demographic, experience, business value and behavioural attributes. The fuzzy expert system assigns a new customer or advisor to the pre-defined categories and provides the corresponding membership values given into the system using fuzzy logic. The author has explained the steps which were followed for the development of the fuzzy expert system. A prototype system has been designed and developed to identify the type of customer and CSA based on the demographic, experience and behavioural attributes. Experimental results are provided and the methodology is validated within the case study approach.
\end{abstract}

Keywords: Fuzzy Expert System, Customer \& Advisor Categorisation, Soft Computing, Contact Centre

\section{Introduction}

In a time of fast growing technology and communication systems, it is very important for the industry and the corporations to develop new customer contact centre (CCC) environment technologies for better customer contact requirements. The integration of customer contact centre into day-to-day organisational operations represents one of the most promising trends in the $21^{\text {st }}$ century economy. The impact is such that contact centres are expected to affect almost all aspects of society from the private sector to public sector in all parts of the world. Whatever the nature or point of contact, customers want a seamless interaction throughout their experience with the company. Customers receive more personalised experience, while the company itself can now provide a consistent message across all customer interactions. Customers want to contact companies at their convenience, using the most convenient means. Good service is now a survival issue, and the competition is on the value that customers receive from their relationship with their suppliers. The companies are aware that it is easier to lose a customer than to gain one; it also knows that it is easier to sell additional services and products to customers who are satisfied with the service provided upon contact; and that a minority of the customer base accounts for the majority of an organisation revenues.

For identifying the type of information required, we needed to develop a fuzzy expert system which would identify the type of customer and advisor based on the demographic, experience, business value and behavioural attributes. Knowing the type of customer and advisor through the categorisation process within an interaction, it can provide better information to the advisor to deal with the customer. A contact centre must both anticipate and react to customers changing needs and demands to achieve strategic customer care. This is turn, has major implications for the kinds of skills, knowledge and competencies of all CCC staff, the systems and the management. The paper is organised as follows: in section 2, we present the related research which is carried out in the design and development of customer and customer advisor categorisation. Section 3 describes the proposed methodology and the design and development of the fuzzy expert system for the categorisation. Section 4 shows the results from the experiments carried out with the model. Section 5 of the paper shows the discussions and suggestions for future work with the part of the research. And finally Section 6 highlights the major conclusions draws from this paper. 


\section{Related Research}

Understanding and adapting to changes of customer behaviour is an important aspect of surviving in a continuously changing environment [Chiu et al. 2002]. Research in understanding customer preferences, known as 'consumer behaviour study', has been the subject of investigation in psychological marketing area for few decades. It is necessary to understand individual customers from designer side, as well as from the customer's side to provide guidance for customers to find what they want. Customer choice of a product depends on explicit requirements, implicit requirements, available options and latent requirements implied by the product [Zeelenberg and Pieters. 2004]. Studies have also shown that complete understanding of service advisor satisfaction requires knowledge of the customers situation before the communication begins [Heckman and Guskey, 1998].

Although research has suggested that customer service advisor (CSA's) performance is critical to create customer satisfaction, little has been done to analyse which employee behaviours influence customer encounter satisfaction and which behaviours influence relationship satisfaction. The performance of a CSA during interactions with customers has been the subject of considerable research, in both sales and service settings. There are five dimensions of CSA's behaviour that influence customer's perceptions: mutual understanding, authenticity, extra attention, competence, and meeting minimum standards [Dolen et al. 2004]. As suggested by Bushey and others for modeling the users can include statements of how the users within a specific user group behave in certain situations or perform certain functions. A system can be designed to accommodate the behavioural diversity of the user groups that most strongly contribute to meeting business goals [Bushey et.al 1999]. The technological change is not simply transforming the methods by which the organisation operates, but is impacting the level of skill and education required by both CSA's and management within the contact centre environment. Many telecoms service sector are subjected to failures in service delivery and better customer satisfaction values because they much depend on customer service advisor (advisors) to deliver service to their customers. Because of the delivery of the service occurs during the interaction between contact advisors and customers, the attitudes and behaviours of advisors can influence customer's perceptions of the services [Hartline and Ferrell, 1996]

Soft computing differs from hard (conventional) computing in that it is tolerant of imprecision, uncertainty and partial truth [Zadeh, 1996]. Soft computing technologies provide an approximate solution to all ill-defined problems and can create user models in an environment, such as behaviour modeling, in which users are not willing to give feedback on their actions and/or designers are not able to fully define all possible interactions. [Martinez et.al 2004] Fuzzy logic has proved useful for developing many practical applications, especially in the field of engineering, as it can handle inexact and vague information. For all the available research been carried out in fuzzy logic and the development of fuzzy expert system for customer modelling, little has been done to categorise the advisor (CSA) within the contact centre domain. Since the expert knowledge captured in If...Then statements is often not naturally true or false, fuzzy sets afford representation of the knowledge in a smaller number of rules, and smooth mapping can be obtained between input and output data [Ngai and Wat, 2003].

Soft computing technologies provide an approximate solution to an ill-defined problem and can create user models in an environment, such as contact centre environment, in which customer willingness to buy or companies prediction towards customer purchase intentions, advisors reaction towards the customers attitude and behaviour, and in turn the customer's behaviour towards the communication with the service advisor within the environment. The elements that a user model captures (goals, plans, preferences, common characteristics of users) can exploit the ability of soft computing of mixing different behaviour and capturing human decision processes in order to implement a system that is more flexible and sensible in relation to user interests. Fuzzy logic provides a mechanism to mimic human decision making that can be used to infer goals and plans; Neural Networks is a flexible mechanism for the representation of common characteristics of a user [Martinez et.al 2004]. It also can be seen as rule based systems that use fuzzy logic in their knowledge base and derive conclusions from user inputs and fuzzy inference process, while fuzzy rules and the membership functions make up the knowledge base of the system. The goal of fuzzy expert system is to take in subjective, partially true facts that are randomly distributed over a sample space, and build a knowledge based ES that will apply to them certain amount of reasoning and aggregation strategies to produce useful decisions [Vadiee et.al 1994].

Mamdani's fuzzy inference method is the most commonly seen fuzzy methodology. Mamdani-type inference, as defined it for the Fuzzy Logic Toolbox, expects the output membership functions to be fuzzy sets. After the aggregation process, there is a fuzzy set for each output variable that needs defuzzification. Sugeno-type systems support this type of model. In general, Sugeno-type systems can be used to model any inference system in which the output membership functions are either linear or constant [Mathworks, 2005] 


\section{$3 \quad$ Proposed Methodology}

The proposed methodology of the research was to develop fuzzy expert system model for categorizing customer and service advisor within contact centres with the use of Matlab fuzzy logic toolbox. The model was developed to assign any customer or advisor within contact centre environment to a pre-determined category determined through the clustering analysis. A fuzzy set allows for the degree of membership of an item in a set to be any real number between 0 and 1, this allows human observations, expressions and expertise to be modeled more closely [Wong et.al 2001] [Zadeh et al 1996]. The steps followed for the development of the expert system model for categorizing customer and service advisor are shown in figure 1 and explained later in the paper.

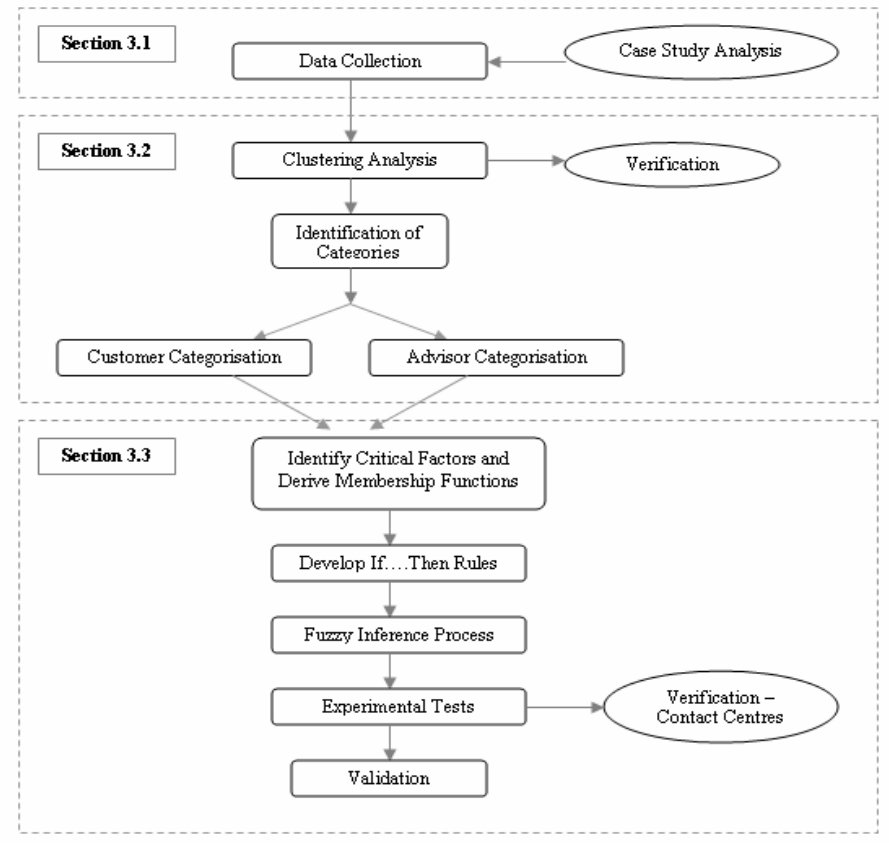

Figure 1 Flow chart for proposed methodology of development of the fuzzy expert system

\subsection{Data Collection}

Data was collected with the help of open set of questionnaires for customer service advisor (CSA) and team leaders/managers with respect to their demographic variables, experience and behavioural variables within five customer contact centre focussing on fault and sales and looking on single to multi profile business customers. A total of 84 advisors were interviewed and assessed, 60 customer calls were heard and monitored, and total of 19 team leaders and managers were interviewed through the questionnaires. For CSA data collection, the author had gone through the questions and also monitored any change in their behaviours during or after the call conversation with the customers at the centre. For customer data collection, the collection was done on the basis of the information provided on the screen of the advisor when the call conversation was in progress, and also the author's hearing to the calls to identify the behavioural aspect of the customer, before the call and once the call was finished. Based on the data collection and the analysis of data the attributes derived for customer and advisor are as follows:

Customer - age, education, financial status, time with company, business value and behavioural analysis Advisor - age, education, experience, previous experience, IT speed, and behavioural analysis

Once the data was collected and analysed it was verified with the team leaders and managers within the contact centres. Based on the verification from the industry the data was structured and analysed using data analysis tool. Through the data analysis tool, the customers and advisor were then grouped in according to the attributes shared among each other. The next stage for the development of the fuzzy expert system model was to identify the categories for customers and advisors through the process of clustering analysis [SPSS Inc]. 


\subsection{Clustering Analysis - Identification of customer and advisor Categorisation}

Classification is a most important and frequently used technique in data mining. It is a process of finding a set of models that describe and distinguish data classes or concepts. Clustering is a method in which we make cluster of objects that are some how similar in characteristics. The aim of the clustering is to provide a grouping of similar records. Based on the data structuring done from the case studies, a data set was designed with 60 samples of customer records and 84 samples (cases) of advisor (CSA's) within the SPSS database. Fuzzy clustering contains two very different areas: the analysis of fuzzy data and the analysis of the crisp data with the help of fuzzy techniques. Fuzzy clustering utilizes fuzzy partitioning to group data such that any given data sample is allowed to belong to several groups with different degrees of similarity bounded within the range of 0 and 1 .

Because of the total number of cases available was of less number and the complexity of the customer data, Two Step Clustering Process within SPSS was used. With two - step cluster analysis, you group observations into clusters based on a nearness criterion. In this process, individual cases are successively combined to form clusters whose centers are far apart. Two step clusters requires only one data pass in the procedure - it passes the data once to find cluster centres (pre cluster stage) and to assign cluster memberships. With two step cluster analysis, we have the flexibility to specify the cluster numbers, specify the maximum number of clusters or let the technique automatically choose the number of clusters. It can handle both continuous and categorical variables and attributes. Based on the samples, clustering analysis was then used to identify the groups of categories which would be derived from the clustering results [Johann et.al 2001] [SPSS Inc].

The selection of different criteria using common linguistic terms for the input variables for age were young, middle age and old; linguistic terms for education were school, college, graduate and professional; for financial status were poor, average and good, for time with company were low, moderate and extensive and so on. For customer selection criteria's used: age were young, middle age and old; for education were school, college, graduate and professional; linguistic terms for financial status were poor, average and good; for time with company were low, moderate and high; for business value were low, medium and high; linguistic terms for positive and negative behaviour were understanding, co-operative, joyful; angry, annoyed and aggressive respectively. Ten different types of experiments were carried out within the two step cluster analysis method ranging from automatic clustering to a maximum of 10 clusters within SPSS. A total of six clusters were derived for customers and advisors. From the clustering analysis the samples of categorisation for customers and customer service advisors (CSA) are:

- Customer Category C1 (Angry Customer) - Female, 18-25, School (education), Poor (financial status), 1-5 yrs (time with company), Low (business value), Angry and Aggressive (behaviour)

- Advisor Category A1 (Novice Advisor) - Female, 18-25 (age), School (education), >1 yrs (experience), Low (IT speed), none (previous exp.), Angry and Unaware (behaviour)

\subsection{Development of Fuzzy Expert System}

Fuzzy logic has proved useful for developing many practical applications, especially in the field of engineering, as it can handle inexact and vague information. The advantages of these systems over conventional production rule based expert systems may be characterised as follows: (a) fuzzy sets symbolise natural language terms used by experts; (b) since the expert knowledge captured in "If....Then" statements is often not naturally true or false, fuzzy sets afford representation of the knowledge in a smaller number of rules; and (c) smooth mapping can be obtained between input and output data. This section discusses the steps followed for the development of the fuzzy expert system for customer and CSA categorisation.

\section{Step 1 - Identify the critical factors and define membership functions and fuzzy sets}

The first step of the process involved the combination of a list of critical factors based on the literature review and indepth interviews with the advisor, team leaders, centre managers and systems expert within the environment. The critical factors were the input variables of the fuzzy ES which were as age, gender, and education, and financial background, time with the company, business value and behaviour from the customer side which would identify the type of category they belong to. 


\begin{tabular}{|ll|}
\hline & \multicolumn{1}{c|}{ Customer Service Advisor (CSA) } \\
1. & Age - young, middle age, old \\
2. & Education - school, college, graduate, professional \\
3. & Experience - novice, medium, senior \\
4. & IT Speed - slow, medium, fast \\
5. & Previous Exp - low, moderate, extensive \\
6. & Positive Behaviour - attentive, friendly, customer \\
& focus \\
7. & Negative Behaviour - unaware, annoyed, angry \\
\hline
\end{tabular}

\begin{tabular}{|ll|}
\hline & \multicolumn{1}{c|}{ Customer } \\
1. & Age - young, middle age, old \\
2. & Education - school, college, graduate, professional \\
3. & Financial Status - poor, average, good \\
4. & Time with Company- low, moderate, high \\
5. & Business Value - low, medium, high \\
6. & $\begin{array}{l}\text { Positive Behaviour - joyful, co-operative, } \\
\text { understanding }\end{array}$ \\
7. & Negative Behaviour - angry, annoyed, aggressive \\
\hline
\end{tabular}

Table 1 Linguistic Terms for Membership Function in the Fuzzy Model

The development of the model was done by author's own understanding for the current contact centre and from the literature studies. Once the selection was done and the model was developed, it was validated with expert judgment from the team leaders at the centre through nine team leaders and managers at three of the case study contact centres. Each linguistic term is defined by a membership function which helps to take the crisp input values and transform them into degree of membership. The simplest membership functions are formed using straight lines. Of these, the simplest is the triangular membership function, and it has the function name trimf. The trapezoidal membership function, trapmf, has a flat top and really is just a truncated triangle curve.

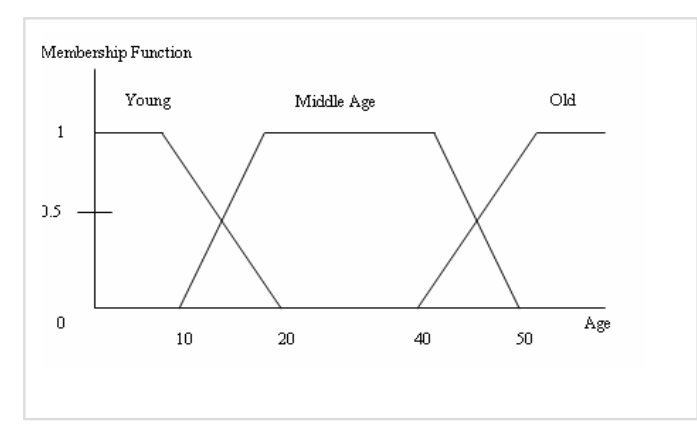

Membership Function for Age

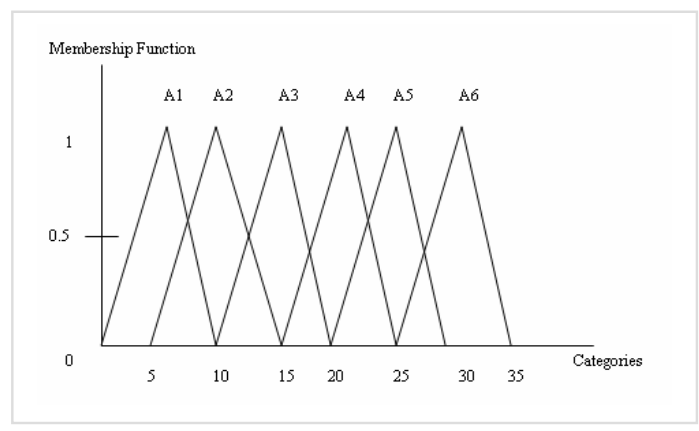

Membership Function for Categories

Figure 2 Sample Membership Functions for Input (Age) and Output (Categories) of the System

\section{Step 2 - Construct the Fuzzy Rules}

Fuzzy ES make decisions and generate output values based on knowledge provided by the designer in the form of IF \{condition\} THEN \{action\} rules. The rule base specifies qualitatively how the output of the system "Category" for the advisor and the customer is determined for various instances of the input variables of Age, Education, Financial Status, and Time with Company, Business Value, Experience, and Behavioral attributes.

\section{If...Then Rules}

Based on the initial results from the clustering of customers and advisors, the author identified the important rules from the results. A sample of the rules derived for the fuzzy logic expert system are shown below and explained later in the section.

\section{Sample Advisor Rules}

\begin{tabular}{|c|c|c|c|c|c|c|c|}
\hline Age & Education & Experience & $\begin{array}{c}\text { Previous } \\
\text { Experience }\end{array}$ & $\begin{array}{c}\text { IT } \\
\text { Speed }\end{array}$ & $\begin{array}{c}\text { Positive } \\
\text { Behaviour }\end{array}$ & $\begin{array}{c}\text { Negative } \\
\text { Behaviour }\end{array}$ & Category \\
\hline Young & School & Novice & Low & Slow & Friendly & Unaware & A1 \\
\hline Middle & Graduate & Medium & Moderate & Medium & Attentive & Annoyed & A3 \\
\hline Old & Profess. & Senior & Extensive & Medium & Focus & None & A5 \\
\hline Young & College & Novice & Moderate & Fast & Focus & Unaware & A6 \\
\hline Young & Graduate & Novice & Low & Fast & Attentive & Annoyed & $\mathrm{A} 2$ \\
\hline Old & Graduate & Senior & Extensive & Fast & Friendly & None & A4 \\
\hline Young & Graduate & Medium & Moderate & Fast & Attentive & None & A2 \\
\hline
\end{tabular}

Table 2 Sample of Advisor Fuzzy If....Then Rules 
Sample Customer Rules

\begin{tabular}{|c|c|c|c|c|c|c|c|}
\hline Age & Education & $\begin{array}{c}\text { Financial } \\
\text { Status }\end{array}$ & $\begin{array}{c}\text { Time with } \\
\text { Company }\end{array}$ & $\begin{array}{c}\text { Business } \\
\text { Value }\end{array}$ & $\begin{array}{c}\text { Positive } \\
\text { Behaviour }\end{array}$ & $\begin{array}{c}\text { Negative } \\
\text { Behaviour }\end{array}$ & Category \\
\hline Young & School & Poor & Low & Low & None & Aggressive & C1 \\
\hline Middle & Graduate & Good & Moderate & Low & None & Annoyed & C2 \\
\hline Old & Graduate & Average & Moderate & Medium & Understanding & Angry & C6 \\
\hline Young & College & Poor & Low & Medium & Co-operative & None & C3 \\
\hline Middle & Professional & Good & Moderate & High & Joyful & None & C5 \\
\hline Old & Professional & Average & High & High & Joyful & Annoyed & C4 \\
\hline Middle & School & Poor & High & Medium & None & Aggressive & C1 \\
\hline
\end{tabular}

Table 3 Sample of Customer Fuzzy If....Then Rules

The above given rules are the sample rules derived for the system. Few rules were written which covered all aspects of the inputs and had a range of output categories for service advisors as A1-A6 categories and C1-C6 categories for customers. The rules within the expert system model were developed from the literature studies and from the understanding the author had developed while doing the case studies and the data collection and analysis phase of the research. Once the rules were derived, the author validated with that of the team leaders from the contact centre, whether the rules were of significance in the real world environment.

\section{$4 \quad$ Experimental Examples and Results}

With respect to the model, the author carried out few experiments with the fuzzy expert system model by changing the input variable values and monitoring the change in the output which showed the change in the category for customer and advisors. Random sampling of 16 experiments was carried out each for customers and advisors. The summary of the experiments which were carried out are shown later in the section 4.1 and 4.2 (refer table 4 and 5). The results derived from the experiments carried out within the expert system model were validated within the contact centre environment with the team leaders and managers. Within the fuzzy expert system model once the membership functions of the input and output variables for customers and advisors were derived, fuzzy if...then rule were written which identified the type of input for customers and advisors. The experiments which were carried out within the model as explained above were done on the basis of the changes made within the rule viewer by changing the input values of the model and observing the output selection of assignment of the categories for customers/advisors.

\subsection{Customer Advisor (CSA) Experiments}

Ex. 1 - If Age = 21.5, Education = 12, Experience = 2, IT Speed = 1.5, Previous Exp = 1.8, Positive Behaviour $=5.5$, Negative Behaviour $=3.8$. Then Advisor Category output is 25 which determines that the category for advisor is A6

Ex.2 - If Age $=30$, Education $=21$, Experience $=4.2$, IT Speed $=5$, Previous Exp $=4$, Positive Behaviour $=1.8$, Negative Behaviour $=5$. Then Advisor Category output is 10 which determines that the category for advisor is A3

The input values for the advisor variables define the type of advisor and output value determines the category for the advisor. For example, the input values in the first experiment is for age $=21.5$, and from our membership functions drawn for the input variables it justifies that the input variable for age is young; education $=12=$ college, experience $=5=5-10 \mathrm{yrs}$, previous $\exp =1.8=$ low and so on. 
Advisor (CSA) Results Summary

\begin{tabular}{|c|c|c|c|c|c|c|c|c|c|c|}
\hline No & Age & $\begin{array}{c}\text { Educa } \\
\text { tion }\end{array}$ & $\begin{array}{c}\text { Exper } \\
\text { ience }\end{array}$ & $\begin{array}{c}\text { Previous } \\
\text { Experience }\end{array}$ & $\begin{array}{c}\text { IT } \\
\text { Speed }\end{array}$ & $\begin{array}{c}\text { Positive } \\
\text { Behaviour }\end{array}$ & $\begin{array}{c}\text { Negative } \\
\text { Behaviour }\end{array}$ & Output & Category & $\begin{array}{c}\text { CC } \\
\text { Validation }\end{array}$ \\
\hline 1 & 21.5 & 12 & 2 & 1.8 & 1.5 & 5.5 & 3.8 & 25 & $\mathrm{~A} 6$ & $\mathrm{~A} 6$ \\
\hline 2 & 30 & 21 & 4.2 & 5 & 4 & 1.8 & 5 & 10 & $\mathrm{~A} 3$ & $\mathrm{~A} 3$ \\
\hline 3 & 20 & 5 & 1 & 0.5 & 1.3 & 1.2 & 1.8 & 5 & $\mathrm{~A} 1$ & $\mathrm{~A} 1$ \\
\hline 4 & 28 & 24.6 & 0 & 1.5 & 3 & 8 & 4 & 5 & $\mathrm{~A} 2$ & $\mathrm{~A} 2$ \\
\hline 5 & 51 & 27 & 8.6 & 5 & 2.8 & 5 & 1.2 & 25 & $\mathrm{~A} 5$ & $\mathrm{~A} 4$ \\
\hline 6 & 43 & 16.5 & 7 & 5.1 & 4.2 & 6 & 0 & 20 & $\mathrm{~A} 4$ & $\mathrm{~A} 4$ \\
\hline 7 & 22.8 & 18 & 2 & 2.1 & 2.5 & 3.2 & 1 & 26.1 & $\mathrm{~A} 6$ & $\mathrm{~A} 2$ \\
\hline 8 & 15 & 2 & 1 & 1 & 0.8 & 7 & 0 & 2.33 & $\mathrm{~A} 1$ & $\mathrm{~A} 1$ \\
\hline
\end{tabular}

Table 4 Experimental Results for Advisor Expert System Model Summary

\subsection{Customer Experimental Results}

Ex. 1 - If Age = 20, Education = 10.2, Financial Status = 2, Time with company = 0.8, Business Value $=4$, Positive Behaviour $=10$, Negative Behaviour $=1$. Then Customer Category output is 5 and the category is $\mathrm{C} 3$ Ex.2 - If Age $=25$, Education $=5$, Financial Status $=3$, Time with company $=5$, Business Value $=2.5$, Positive Behaviour $=1.2$, Negative Behaviour $=5$. Then Customer Category is $\mathrm{C} 1$

Customer Results Summary

\begin{tabular}{|c|c|c|c|c|c|c|c|c|c|c|}
\hline $\begin{array}{c}\mathbf{N} \\
\mathbf{o}\end{array}$ & Age & $\begin{array}{c}\text { Educa } \\
\text { tion }\end{array}$ & $\begin{array}{c}\text { Financial } \\
\text { Status }\end{array}$ & $\begin{array}{c}\text { Time with } \\
\text { Company }\end{array}$ & $\begin{array}{c}\text { Business } \\
\text { Value }\end{array}$ & $\begin{array}{c}\text { Positive } \\
\text { Behaviour }\end{array}$ & $\begin{array}{c}\text { Negative } \\
\text { Behaviour }\end{array}$ & $\begin{array}{c}\text { Output } \\
\text { Value }\end{array}$ & $\begin{array}{c}\text { Categ } \\
\text { ory }\end{array}$ & $\begin{array}{c}\text { CC } \\
\text { Validation }\end{array}$ \\
\hline 1 & 20 & 10.2 & 2 & 0.8 & 4 & 10 & 1 & 15 & $\mathrm{C} 3$ & $\mathrm{C} 3$ \\
\hline 2 & 25 & 5 & 3 & 5 & 2.5 & 1.2 & 5 & 5 & $\mathrm{C} 1$ & $\mathrm{C} 1$ \\
\hline 3 & 30 & 7 & 8.9 & 9 & 6.8 & 5 & 0 & 25 & $\mathrm{C} 5$ & $\mathrm{C} 5$ \\
\hline 4 & 36 & 16.5 & 6.5 & 4.5 & 5 & 6.2 & 10 & 10 & $\mathrm{C} 2$ & $\mathrm{C} 2$ \\
\hline 5 & 28 & 10.7 & 0 & 0 & 5 & 10 & 2.1 & 15 & $\mathrm{C} 3$ & $\mathrm{C} 3$ \\
\hline 6 & 40 & 25 & 5 & 10 & 8.5 & 9 & 0.4 & 20 & $\mathrm{C} 4$ & $\mathrm{C} 6$ \\
\hline 7 & 50 & 10 & 4.3 & 6.5 & 0 & 7 & 3 & 30 & $\mathrm{C} 6$ & $\mathrm{C} 4$ \\
\hline 8 & 18 & 1.2 & 1.5 & 3 & 1.2 & 1.2 & 8 & 5 & $\mathrm{C} 1$ & $\mathrm{C} 1$ \\
\hline
\end{tabular}

Table 5 Experimental Results for Customer Expert System Model Summary

Based on the model, the author identified that the results derived from the model, assigned a customer with the predetermined category which were derived from the clustering. These results were also validated with the team leaders at the contact centre to verify that the given selection of the pre-determined categories for customer was properly justified. The discussion for few of the experiments related to the customer and the service advisor as explained later in discussions and future research section of the paper.

\subsection{Validation}

The information and the results from the model were verified within industry expert judgment through team leaders and managers at three of the contact centres where the case studies were carried out. A total of nine team leaders and managers were interviewed with the help of an open set questionnaire, showing the categories derived and the assignment of a particular customer or advisor to these categories through the help of the fuzzy expert system tool developed. The team leaders at the contact centre were shown the possible combinations of the customer and advisor categories, and on what basis these categories were derived. The results of the validation are shown earlier in section 4.1 and 4.2 explaining to which of the customer and advisor categorizations the team leaders correspond to be true and satisfied with the experimental results. 


\section{$5 \quad$ Discussions and Future Research}

The authors have demonstrated the steps which were followed for the development of a fuzzy expert system to assign the customer and advisor to the pre-determined category. The experimental results in table (4) and (5) shows that $80 \%$ of results are as expected, and were assigning a particular customer and advisor to the categories which were derived from clustering. Based on experiment 5, the expert system assigned category A5 to the advisor. However from validation with team leaders it revealed that the category should be A4. On the basis of the validation the changes were made with respect to behavioural attributes from friendly behaviour to customer focus behaviour. Experiment 7 revels that the expert system assigned category A6, which on further validation with team leaders at the contact centres fall into A2 category. The reasons for this swift change in selection of category were due to:

a) Education level to be high.

b) Positive behaviour to be attentive.

c) Less amount of negative behaviour.

The rules were fine tuned to predict A2 category and share characteristics of that category.

For customer categorisation, the results from the expert system for experiment 6 and 7 did not match that to the validation from the team leaders at CC (table 5). Necessary modifications were carried out within the expert system to assign a category to customer to match with the validation results from the team leaders. As seen in experiment 6 , the changes made were education level was changed from graduate to college level to assign customer with C6 category. Experiment 7 revealed that expert system assigned C6 category which on further validation fall into $\mathrm{C} 4$ category. The changes made within the expert system were:

(a) Customer time within company.

(b) Positive attitude towards the advisor and

(c) Less amount of negative attitude shown from the customer.

The next step of this research is to develop an information required framework which would identify the type of information required by the advisor to serve the customer.

This paper discusses the design and development of a fuzzy expert system methodology that incorporates behavioural attributes along with demographic, experience and business value attributes for customers and advisor categorisation within contact centre environment. On the basis of assignment of any customer or advisor through the expert system tool to that of pre-determined category we can identify the type of caller (customer) and the random advisor assigned to that customer. Once the categorisation of customer and advisor is finished, we can then provide the advisor with the minimum amount of information which is required to be displayed on the screen to help the advisor to provide better customer satisfaction towards the customer. The results from the expert system model are going to be used in further implementation of the prototype tool on the type of information requirement on the possible \{customer, advisor \} combinations that can be implemented within contact centre environment.

\section{Conclusions}

This paper is focused on the development of methodology for designing fuzzy expert system for customer and advisor (CSA) categorisation within contact centre environment. The authors identified the initial attributes to be used within the model for customer and advisor through five case studies carried out at the customer contact centres within UK ranging from telecoms to help desk and government; and 84 customer advisor and 60 customers. Based on the data collection and analysis of data, the author identified the attributes for advisor as age, education, experience, previous experience, IT speed, and behavioural attributes; and for customers attributes such as age, education, and time with the company, business value, and behaviours respectively. Based on the initial clustering results, the authors then derived the initial categorisation for customers and advisors.

A fuzzy expert system was developed to assign any customer or advisor to that of the pre-determined category from the clustering analysis. Experimental tests were carried out to check the assignment of the customer and advisor to the categories derived from the clustering analysis were same and related to each other. The results showed that over eighty percent of the time the assignment from the expert system for the categorisation of the customer and advisor was correct; which was validated with the team leaders at the case study contact centres. A proper methodology was followed for the development of the fuzzy expert system for categorisation of customer and service advisors within the contact centre and the results from the model were validated within the real contact centre environment. Future directions that can be carried out with this research were explained in the section five. The next step through this research is to develop an information required framework which would identify the type of information required by the advisor to serve the customer on the basis of the customer and advisor categorisation. 


\section{Acknowledgements}

The author wish to acknowledge the support of the Engineering and Physical Sciences Research Council (EPSRC), BT Telecommunication Plc, and Decision Engineering Group (DEG) Cranfield University; UK.

\section{References}

Bushey, R.; Mauney, Jennifer. M and Deelman, Thomas (1999). The development of behaviour-based user models for a computer system. $7^{\text {th }}$ International Conference on User Modeling (UM99). 20 $0^{\text {th }}-24^{\text {th }}$ June 1999 . Banff Centre, Banff. Canada. Pp 109-118. Link - www.cs.usask.ca/UM99/Proc/bushey.pdf

Chiu, Chaochang (2002). A case based customer classification approach for direct marketing. Expert Systems with Applications. Vol 22 (2002). Pp 163-168. Elsevier Science Ltd.

Dolen, Willemijn van; Ruyter, Ko de and Lemmink, Jos (2004). An empirical assessment of the influence of customer emotions and contact employee performance on encounter and relationship satisfaction. Journal of Business Research. Vol 57 (2004). Pp 437-444. Elsevier Science Ltd.

Goff, Brent. G; Boles, James. S; Bellenger, Danny. N and Stojack, Carrie (1997). The influence of salesperson selling behaviours on customer satisfaction with products. Journal of Retailing. Vol 32. No 2. pp 171-183.

Harline, Michael. D and Ferrell, O. C (1996). The management of customer contact service employees: an empirical investigation. Journal of Marketing. Vol 60 (1996). Pp 52-70.

Heckman, Robert and Guskey, Audrey (1998). Sources of customer satisfaction and dissatisfaction with information technology help desks. Journal of Market Focused Management. Vol 3. pp 59-89.

$\mathrm{Hu}$, Tung-Lai and Sheu, Jiuh-Biing (2003). A fuzzy based customer classification method for demand responsive logistical distribution operations. Fuzzy Sets and Systems. Vol 139. No.2 October 2003. PP 431-450

Johann Bacher, Knut Wenzig and Melanie Vogler. (2001). SPSS Two Step Cluster - A first evaluation. http://www.statisticalinnovations.com/products/twostep.pdf

Martinez, F.E; Magoulas G.D.; Chen S., and Macredie R. (2004). Recent soft computing approaches to user modeling in Adaptive Hypermedia. Proceedings of 3rd Int. Conference Adaptive Hypermedia-AH 2004, Eindhoven, The Netherlands, Aug. 2004, Lecture Notes in Computer Science, vol. 3137, Springer, 104-113 http://www.dcs.bbk.ac.uk/ gmagoulas/AH04-Frias-Magoulas-Chen-Macredie.pdf

Mathworks Fuzzy Logic Toolbox. Model based design software. Available at: http://www.mathworks.com/products/fuzzylogic/ (accessed on: 15/01/05)

Ngai, E.W.T and Wat, F.K.T (2003). Design and development of a fuzzy expert system for hotel selection. The International Journal of Management Science. Omega. Vol 31. PP 275-86. Elsevier Science.

Song, Hee. Seok; Kim, Jae. Kyeong and Kim, Soung. Hie (2001). Mining the change of customer behaviour in an Internet shopping mall. Expert Systems and Applications. Vol 21. pp 157-168. Elsevier Science Ltd.

SPSS Inc (2005). Statistics \& Data Mining Software: SPSS. Available at: http://www.spss.com/ (accessed 03/04/2005).

Swinyard, William.R (2003). The effects of salesperson mood, shopper behaviour and store type on customer service. Journal of Retailing and Consumer Services. Vol 10 (2003). Pp 323-333. Elsevier Science Ltd.

Wong, K.M (2001). Data mining using fuzzy theory for customer relationship management. $4^{\text {th }}$ Western Australian Workshop on Information Systems Research (WAWISR.2001). 26 ${ }^{\text {th }}$ Nov 2001. Perth, Australia. http://wawisr01.uwa.edu.au/WAWISR.pdf .

Zadeh, L. (1996). The role of soft computing and fuzzy logic in conception, design and deployment of intelligent systems. Proceedings International Workshop on Soft Computing in Industry, Muroran, Japan, April 1996, pp $136-137$.

Zeelenberg, Marcel and Pieters, Rik (2004). Beyond valence in customer dissatisfaction: A review and new findings on behavioural responses to regret and disappointment in failed services. Journal of Business Research. Vol 57 (2004). Pp 445-455. Elsevier Science Ltd. 